REVIEW

\title{
PREDICTIVE FACTORS FOR THE RESPONSE OF LOCAL AND ADVANCED RECTAL CANCER TO NEOADJUVANT RADIOCHEMOTHERAPY
}

\author{
Ana Constantin ${ }^{1}$, P. Papuc ${ }^{1}$, D.N. Păduraru ${ }^{1,2}$ \\ ${ }^{1}$ The University of Medicine and Pharmacy "Carol Davila", Bucharest, Romania \\ ${ }^{2}$ The General Surgery and Emergency Clinic III - The University Emergency Hospital of Bucharest, Romania \\ Corresponding author: Dan Nicolae Păduraru \\ Phone no. 0040744756443 \\ E-mail: dan.paduraru.nicolae@gmail.com
}

\begin{abstract}
Rectal cancer is an aggressive form of cancer whose treatment follows the next steps: neoadjuvant therapy, surgical excision, and postoperative therapy.Currently, they are trying to identify some predictive factors related to the response to neoadjuvant therapy. Among these factors there are: the characteristics of the tumor - according to the TNM classification; the character of the tumor by monitoring Ki67 protein and mRNA, by thymidylate synthase and EGF determination, as well as independent traits such as age and sex. To the extent in which the factors mentioned anticipate the uselessness of the neoadjuvant therapy, it can be excluded from the treatment plan, which implicitly implies benefits such as reduction of the time interval elapsed until the surgical intervention, and also reduction in the duration and cost of treatment. There are also short-term benefits. The patient will no longer face deterioration in his psychological status and decreased immunity, or other adverse effects of radiochemotherapy.
\end{abstract}

Keywords: rectal cancer, neoadjuvant radiochemotherapy

\section{Introduction}

Colorectal cancer (CRC) is the third in frequency in the world with an estimation of 72,090 new cases / year in men and 70,480 in women in the United States. It is also the third leading cause of death due to neoplastic processes, with an estimated figure of 26,580 and 24,790 deaths / year for men and women respectively [1].

In Romania, the frequency of $\mathrm{CRC}$ is increasing rapidly (doubling its values in terms of incidence and mortality over the past 20 years), reaching an incidence of $17.74 \% 000$ inhabitants. In 2006, there were 8,240 new cases which made Romania one of the countries with an average incidence of the disease.

After the year 2000, colorectal cancer has become the second leading cause of death (after bronchopulmonary cancer), but ahead of gastric cancer, with a total figure of 4,150 deaths in 2002 (19.05\% 000 inhabitants) and 4,860 deaths in 2006. The ratio men / women is 1.3. Less than $3 \%$ of the cases occur in people aged below 40. The incidence increases rapidly over the age of 45 and doubles with each decade of life.

Rectal cancer represents $30-40 \%$ of all colorectal neoplasms and, at the same time, one third of the number of cases of cancer diagnosed annually worldwide, in both men and women. 
The standard treatment for rectal cancer is radical surgery whose goal is to excise the tumor with wide limits and regional lymphadenectomy. Multimodal therapy has recently developed and comes to support the surgical approach. It implies the association of chemoradiotherapy and adjuvant therapy. The multimodal therapy manages to revolutionize the previous approach by gathering the exponents of all therapeutic approachces. Its goal is cytoreduction, which brings about two notable advantages: it permits the resection of low tumors and tumor downgrading consecutive to the surgical intervention. The treatment of tumors situated at less than 6-7 cm from the anal line involves the amputation of the rectum, with all the discomfort the procedure might bring by removing the sphincterian apparatus; those situated at more that $6-7 \mathrm{~cm}$ from the anal help concluding the case through resection. The neoadjuvant therapy can convert a first-type tumor into a second-type one, thus avoiding the amputation of the rectum.

The therapeutic sessions can take place both prior and after surgery. There are two possible scenarios with strict reference to neoadjuvant therapy:

- The first one refers to the situation in which the therapeutic scheme proves efficient and the surgical excision becomes easier due to the reduced dimensions of the tumor.

- The second scenario involves an increased resistance to radiochemotherapy, which allows the evolution of the tumor.

It is clear that in the latter case, given the disadvantages involved by the neoadjuvant therapy and the fact that the tumor is increasing in dimensions, it would be preferable that the surgical intervention were scheduled as soon as possible, in which case the neoadjuvant therapy might be perceived useful.

In addition to the variability of the response of rectal tumors to neoadjuvant therapy, radiation therapy isn't an easily accessible tool in Romania.

Therefore, it is legitimate to ask questions like:

- "How and if we can anticipate the evolution of rectal cancer after neoadjuvant therapy?"

- "Which are the patients that would greatly benefit from radiotherapy in order to make the delay of the assumingly curative surgical intervention worthy?"

- "When and for which patients with advanced local tumors of the rectum should we perform a continuous colostomy before seeing the radiotherapist-oncologist?"

The experience full of unexpected developments made us ask ourselves these questions and make an extremely serious analysis of the variability of responses to neoadjuvant therapy in rectal cancer.

Although major steps have been made to identify the biomarkers with predictive and prognostic properties and not all the studies have been able to identify such factors, we have decided to do a review of the specialty literature and to mark some pathways and express some opinions on the topic.

For a better understanding, we must stress the difference between the following two terms - predictive and prognostic. The first approaches the subject qualitatively; a predictive factor indicates whether the therapy is effective or not, and the second provides a quantitative description; a prognostic factor assesses the usefulness of a certain type of therapy.

This paper aims to identify the main predictive factors (a predictive factor is any parameter that evaluates the response or lack of response to a specific treatment), which might be included in the development of an algorhythm for predicting the evolution of rectal tumors, in order to establish a more efficient treatment regimen.

For teaching purposes, the approach of the theme will be made following several key points regarding the response of the rectal tumor to neoadjuvant therapy:

1. The general characteristics of tumors

2. The tumor character

3. Independent factors

1. We can use the TNM classification to refer to the general characteristics of tumors. This classification quantifies the severity of the tumor from several perspectives.

- " $\mathrm{T}$ " refers to the assessment of tumor expansion. For its postoperative evaluation, investigation measures are required such as echoendoscopy, MRI, CT scan. The features described by this type of investigation have a minimum impact upon the response of the 
tumors to the neoadjuvant therapy. We said minimum because, although found in stages defined as locally advanced, the small-sized tumors react better to the neoadjuvant therapeutic scheme.

- The second element in TNM classification and also one of the most effective prognostic and predictive factors of response to neoadjuvant treatment is the degree of lymph node invasion. As in the previous case, there is not a single type of investigation used for evaluating and monitoring this parameter. Among the most frequently used ones there are: echoendoscopy, pelvic MRI, CT scan, PETSCAN. The results of the multiple research confirm that tumors in the early stages (NO) are marked by a favorable evolution, responding positively to the neoadjuvant treatment in a ratio of $43 \%$.The tumors that have already comprised one or two limph node clusters - N1-N2 - are less receptive and have a favorable response to the neoadjuvant treatment in only $9 \%$ of the cases.

Therefore, the ganglionic invasion in case of rectal cancer is not only a prognostic factor but also a predictive one related to the response to neoadjuvant therapy.

- M-the last parameter in the TNM classification concerns the degree of metastasis, indicating the number of metastases and whether they can be local or distant. The higer the degree of metastasis, the fewer the chances of curing.

2. Tumors are generally the result of uncontrolled proliferation of some altered cells. The rate of cellular proliferation is a key factor in the development of the tumor. This is influenced by not stimulating and inhibiting factors, more exactly by their absence or reduced expression.

Disturbances may occur both in cellular division and genetic expression and also among nucleotide fragments.

- Ki67 protein can be identified in all the stages of the cellular cycle, except the Go phase, which is the relaxation phase. The selective presence of this protein in patients with neoplasms makes it be defined as a marker of choice in monitoring the multiplying rate of neoplastic formations. Its presence in high amounts clearly indicates the intensification of cellular replication, and consequently the tumor has a rapid and a very aggressive evolution. Antibodies are synthesized against this protein. Their dosage is useful because it characterizes the degree and manner in which the body opposes cellular proliferation. Therefore, an increased value of these antibodies provides a good prognosis and encourages the introduction of neoadjuvant radio-chemotherapy therapy in the scheme of treatment.

- In addition to a strict assessment of cellular division, the expression of genes must be brought into discussion. The exploitation of a minimal imbalance may generate consequences with a major impact on the response to neoadjuvant therapy. Looking from this perspective, we can distinguish tumors that are rapidly evolving and tumors with a slower evolution. MicroRNA is a short RNA fragment which doesn't present a coding capacity, which plays a role in post-translational modulation of some genes by inactivating the corresponding messenger RNA. Recently, it has been found that it acts upon the genes that encode a variety of properties such as: tumor invasion of adjacent tissues, lymphatic and hematogenous dissemination and resistance to various forms of therapy. Moreover, these genes also have an influence on the dynamics of suppressor genes and oncogenes. There are several subtypes of miARN, each of which having its characteristic targets. Mutation in miARN structures is a very important factor in the development of several types of cancer, including rectal cancer.

We mention a research study whose outcome is extremely interesting. After outling the RNA profile, two groups were formed: one group with increased responsiveness to therapy and good results and another one characterized by an increased resistance to the neoadjuvant therapy. The two groups were analyzed in parallel in terms of response to neoadjuvant therapy, concluding upon the excessive presence of some types of RNA. The unresponsive group was marked by an exaggerated expression of MIR-190b, 29b2 MIR, and MIR-215. We might conclude by stating that the presence of these markers anticipates an unfavorable response to therapy. Reversely, the presence of MIR196b, MIR-450a, MIR 450b-5p, and let-7e might foreshadow a favorable response. 
It is certain that microRNAs are important both in the occurrence of tumor formation and as a predictive factor and it should not be ignored.

- The modulator of cellular apoptosis - Bax, encoded by the Bax gene acts as a natural limiting factor of cell replication. Bax is a protein involved in the activation of cellular apoptosis by opening the voltage-dependent anion channels (VDAC), thus generating a consecutive flow of ions. This change causes the loss of mitochondrial membrane potential and the release of cytochrome $\mathrm{C}$. The increased expression of this protein predicts a good evolution since its presence is correlated with a favorable response to neoadjuvant therapy.

- The stimulating factors of cellular proliferation are absolutely essential for the normal development of the body and for the processes of tissular recovery, but the process must be controlled. The overexpression of such factors leads to the occurrence of neoplastic formations. The carcinoembryonic antigen is a marker, found on the cellular membrane, whose function isn't completely known. It acts as a suppressor by stimulating $\mathrm{T}$ suppressor lymphocytes and macrophages. The carcinoembryonic antigen is an oncofetal gene, thus synthesized during the embryonic stage, whose production is suppressed at birth and whose values during adulthood are much lower. There are differences between the values recorded in smokers and nonsmokers. The nonsmokers may take dosages of up to $3 \mathrm{ng} / \mathrm{ml}$ and the smokers up to $5 \mathrm{ng} / \mathrm{ml}$. The reduced expression of the carcinoembryonic antigen is associated with a higher likelihood of positive response to neoadjuvant therapy. It is important to notice that this correlation is valid only in nonsmoking patients. In smokers, the carcinoembryonic antigen is not relevant as a predictive factor for the response to neoadjuvant therapy in rectal cancers.

- EGF is a factor responsible for the stimulation of cellular with an endodermal, ectodermal and mesodermal origin, and it may sometimes act as a factor of cellular differentiation. Moreover, from a structural point of view, it is the receptor corresponding to EGF-REGF. Numerous studies have demonstrated that the absence of responsiveness of rectal tumors to neoadjuvant treatment is directly proportional with the expression rate of these receptors at a cellular level. Moreover, the presence of a small number of such receptors has been associated with a reduced risk of metastasis.

- Not only markers relevant for the economy of cellular processes are worth mentioning. There are also cellular components that manage to directly influence the effectiveness of drugs used in oncotherapy. In addition to the physiological role of a catalyst, the enzymatic structures may acquire new features in a therapeutical context. Thymidylate synthase is an enzyme responsible for procuring the thiamine structure necessary in the synthesis of DNA structures. At the same time, it converts 5-FU, an active compound used in chemotherapy. The newly-formed compound is associated with thymidylate synthase and inhibits cell synthesis, which enhances the therapeutic effect of neoadjuvant chemotherapy.

Given the development of this process, the use of neoadjuvant radiochemotherapy is indicated in patients with a large amount of thymidylate synthase (4). Complementarily, a reduced expression of these factors is associated with an unfavorable prognosis.

3. The third perspective suggested for discussion was that of independent characteristics which comprise the age and sex. The approach concerning this aspect was quite difficult and although hypotheses stating that the elderly are more responsive to neoadjuvant therapy have been made, the conclusion remained that these two criteria - age and sex are both irrelevant.

All the factors mentioned so far are independent factors. There are also factors that have allowed us to make correlations with the development of the tumor after neoadjuvant therapy, that are not independent. 78 Grp is a protein that belongs to the heat shock protein superfamily (Hsp70). Among other roles, Hsp70 protects the cell from apoptosis. Another example is that of microvascular density and VEGF (vascular epithelial growth factor). The unfavorable response of colon cancer to the 
neoadjuvant therapy is expected to have higher than normal values of these elements.

\section{Discussions}

The multimodal therapy comprises chemotherapy and radiation therapy, whose effectiveness may increase if associated with different factors, such as radiotherapy associated with chemical substances.

Recent studies have exploited the effect of thymidylate synthase from several perspectives. It has been proven that this enzyme is useful in enhancing the effect of chemotherapy. However there is no correlation between the high level of thymidylate synthase and the efficiency of radiotherapy. It is worth mentioning that the quantitative properties of this enzyme maximize the potential of therapeutic combinations. For example, the stem cells with a higher content of thymidylate synthase are more susceptible to the cytotoxic effect of Oxalipatin. Moreover, it is a marker to be taken into account when it is noticed that the therapy with 5-FU has a low efficiency. When associating Oxalipatin with $5 \mathrm{FU}$ the presence in high amounts of this enzyme becomes relevant. Determination of this enzyme implies using reactions based on immune responses. An interesting aspect is that in most studies, patients are divided only into two groups - one presenting the marker in a small quantity, and the other one presenting it in an increased amount. This aspect is a significant disadvantage because it becomes difficult to estimate which is the range within whose limits the conversion from one category to another is likely, so that the quantitification of thymidylate synthase be included in an algorhythm aimed at predicting the evolution and stating the prognosis. The results were contradictory in some cases because the importance of the enzyme is influenced by the way in which the mediciation (5FU) is administrated, an aspect that the surgeon should be informed of and that he should take into account. If administered in the bolus, it has a stronger effect on the RNA and if administered continuously is considerably affects the TS.

The enzyme was considered a prognostic and predictive factor for postoperative therapy. Still, it is worth mentioning that the determination of the enzyme in the tumor might not be compatible with the value obtained from the determination of the enzyme at the level of the invaded nodes or metastases.

\section{Conclusions}

- The large number of predictive markers available at the moment in the stage of preoperative evaluation represents extremely important elements that might form the basis of prediction algorhythm needed for the evaluation of patients with locally-advanced rectal cancer.

- The high variability of predictive factors highlighted so far is essential in ensuring an effective redistribution of patients, depending on the need for application of neoadjuvant therapy before surgery.

- The elements associated with tumor progression during the neoadjuvant therapy are the most diverse:

- The parameters of the cellular cycle - the presence of large amounts of the modulators of Bax cellular apoptosis, Ki67 protein and thiamine synthase enzyme, are usually associated with a good prognosis.

- The reduced expression of some factors opposing cellular proliferation, such as carcinoembryonic antigen, has proven to decrease the efficiency of the neoadjuvant radio-chemotherapy.

- The potential that this algorhythm has is a huge one. It could represent a revolution in the field of therapeutic standardization of the neoplasms located in the rectum through the application of some personalized schemes of treatment for each pair of the type tumor-patient.

\section{References}

[1] Adjuvant and neoadjuvant treatment for rectal cancer, colon cancer, and non-small-cell lung cancer in older patients, R. Rahal, MBA, G. Porter, $\mathrm{MD},{ }^{\dagger}$ T. Forte, MSc, ${ }^{*}$ S. Fung, MSc, ${ }^{*}$ H. Bryant, MD $\mathrm{PhD},{ }^{* *}$ and Collaboration with the System Performance Steering Committee and the Technical Working Group

[2] National Comprehensive Cancer Network (NCCN) NCCN Clinical Practice Guidelines in 
Oncology: Rectal Cancer. Fort Washington, PA: NCCN; 2014. Ver. 3.2014

[3] Sanoff HK, Carpenter WR, Stürmer T, et al. Effect of adjuvant chemotherapy on survival of patients with stageIII colon cancer diagnosed after age 75 years. J Clin Oncol. 2012;30:2624-34. doi: 10.1200/JCO.2011.41.1140.

[4] Pasetto LM, Friso ML, Pucciarelli S, et al. Rectal cancer neoadjuvant treatment in elderly patients. Anticancer Res. 2006;26:3913-23.

[5] Sebag-Montefiore D, Stephens RJ, Steele R, Monson J, Grieve R, Khanna S, Quirke P, Couture J, de Metz C, Myint AS, et al. Preoperative radiotherapy versus selective postoperative chemoradiotherapy in patients with rectal cancer (MRC CR07 and NCIC-CTG C016): a multicentre, randomised trial. Lancet. 2009;373:811-820.

[6] Sauer R, Becker H, Hohenberger W, Rödel C, Wittekind C, Fietkau R, Martus P, Tschmelitsch J,
Hager E, Hess CF, et al. Preoperative versus postoperative chemoradiotherapy for rectal cancer. N Engl J Med.2004;351:1731-1740.

[7] Du CZ, Xue WC, Cai Y, Li M, Gu J. Lymphovascular invasion in rectal cancer following neoadjuvant radiotherapy: a retrospective cohort study. World J Gastroenterol. 2009;15:3793-3798.

[8] Wang L, Gu J. Risk factors for symptomatic anastomotic leakage after low anterior resection for rectal cancer with $30 \mathrm{~Gy} / 10 \mathrm{f} / 2 \mathrm{w}$ preoperative radiotherapy. World J Surg. 2010;34:1080-1085.

[9] Kim NK, Baik SH, Min BS, Pyo HR, Choi YJ, Kim H, Seong J, Keum KC, Rha SY, Chung HC. A comparative study of volumetric analysis, histopathologic downstaging, and tumor regression grade in evaluating tumor response in locally advanced rectal cancer following preoperative chemoradiation. Int J Radiat Oncol Biol Phys. 2007;67:204-210. 